\title{
Framing a satellite based asset tracking (SPARTACUS) within smart city technology
}

\author{
Fabio Casciati $^{1}$, Sara Casciati ${ }^{2}$, Clemente Fuggini ${ }^{3}$, Lucia Faravelli ${ }^{1}$, Ivan Tesfai ${ }^{3}$ and Michele \\ Vece $^{1^{*}}$ \\ ${ }^{1}$ DICAr, University of Pavia, Via Ferrata 3, Pavia 27100, Italy \\ ${ }^{2}$ DICAr, School of Architecture, University of Catania at Siracusa, P.za Federico di Svevia, Siracusa 96100, Italy \\ ${ }^{3}$ D’Appolonia S.p.A., Piazza Duca D’Aosta 14, Milano 20124, Italy
}

\begin{abstract}
Smart city" is a term currently used to denote cities moved by the opportunity to enhance the quality of life and the security of their citizens. Attention is here focused on the concept that the development of smart cities can also be achieved by improving the efficiency of civil infrastructures through a real-time monitoring. This is the specific target of the European Union FP7 project (SPARTACUS), moved from the parallel chance to develop industry pull applications for the European EGNOS and GALILEO satellite systems. In this paper, laboratory tests are carried out to provide specific devices the ability to run while satisfying the requirements of the incoming GALILEO system. Moreover, some of the targets are achieved within the current GPS system. An extensive experimental campaign is offered to validate the units in such a scenario.
\end{abstract}

Keywords: smart cities, asset tracking, security, positioning, data transmission, GNSS

*Correspondence to: Michele Vece, DICAr, University of Pavia, Via Ferrata 3, Pavia 27100, Italy; Email: miche.vece@gmail.com

Received: October 30, 2016; Accepted: November 17, 2016; Published Online: December 30, 2016

Citation: Casciati F, Casciati S, Fuggini C, et al., 2016, Framing a satellite based asset tracking (SPARTACUS) within smart city technology. Journal of Smart Cities, vol.2(2): 40-48. http://dx.doi.org/10.26789/JSC.2016.02.003.

\section{Introduction}

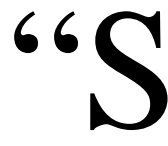
mart city" is a term recently introduced to denote a city that uses Information and Communication Technologies (ICTs) in order to enhance the quality of life of the citizens and to contribute to its sustainable development. Smart city initiatives attracted the attention of many researchers and politicians on issues such as mobility, security, environment, or any combination of them, since they are driven by the goal of serving citizens in a better way ${ }^{[1]}$. Moreover, smart cities facilitate the government to monitor, understand, analyze, and plan the city in order to improve the equity, efficiency, and quality of life ${ }^{[2,3]}$.

The attention of the research activity summarized in this paper is focused on the concept that the smart city development provides opportunities to enhance the quality of civil infrastructures as well. Indeed, smart cities lead to a significant evolution of roads, bridges, tunnels, and rail through a real-time monitoring for maximizing the services security of the residents while optimizing the use of resources.

Within this framework, special care is paid to the European Union FP7 project named SPARTACUS (Satellite Based Asset Tracking for Supporting Emergency Management in Crisis Operations), currently in progress $^{[4]}$. It is moved from the parallel opportunity to develop industry pull applications and solutions for the European EGNOS and GALILEO satellite systems. Within the project, satellite-based positioning units, also mounting Inertial Navigation Systems (INS),

Framing a satellite based asset tracking (SPARTACUS) within smart city technology. (C) 2016 Fabio Casciati, et al. This is an Open Access article distributed under the terms of the Creative Commons Attribution-NonCommercial 4.0 International License (http://creativecommons.org/licenses/bync/4.0/), permitting all non-commercial use, distribution, and reproduction in any medium, provided the original work is properly cited. 
are tested in order to track critical transport assets, relief goods distribution, and first responders in disaster management operations ${ }^{[5]}$. The idea is not new, but its extensive use in full-scale tests makes the dissemination of interest.

One of the main challenges is related to the security issues. Indeed, smart cities require access to cost effective, high performance security services, and sophisticated control systems, which ensure the higher network reliability. Therefore, it is important to instil awareness that digital infrastructures may be subjected to information thefts and thus results in an increase in cyber-crimes. Potential can be undermined in case of unauthorized electronic access, and technological issues can arise from hardware/software incompatibility because everything in smart cities has to be automated and any fragmentation may lead to serious failures and accidents.

This paper starts from the current state of the art in order to understand the need of the proposed system. After an overview of the adopted system architecture, the integration of some devices is discussed. A broad experimental campaign of field tests follows to validate the units in the presented scenario. Simulations of test results are also carried out in order to provide an already running system exploiting features of the incoming GALILEO.

\section{Governing Relations}

Before detailing the innovative aspects of the solution proposed within the abovementioned FP7 project (SPARTACUS), it is convenient to show the current drawbacks faced daily by rescue teams, which need to be informed in time when a catastrophic event occurs.

\subsection{Transportation and Security}

Throughout the world, the transport of goods is greatly increased in terms of volume and variety over the last decades. This growth is the main cause of some substantial costs such as cumulative congestion levels and associated environmental pollution, but especially in term of accidents risk and time wastage during traveling $^{[6]}$. Traditional approaches failed to solve issues like these.

New ways are currently under investigation with the aims to develop an effective system able to increase network efficiency, without compromising safty, and to provide accurate and reliable data for maintenance and planning purposes in case of emergency.
A real case is used to highlight current operational challenges and technology gaps occurring in a disaster situation. It is used to emphasize how the SPARTACUS architecture has to operate.

Trains in Italy frequently use the rail system in the Piedmont region, where several areas are exposed to erosion. During the winter period, the situation is aggravated by severe weather. In late February 2016, it was reported that there was an alert status due to occasional storms, abundant rains, and flooding until, on February 28, a passenger train from Biella to Novara derailed after a landslide from the rock overlying the retaining wall that caused its collapse (Figure 1). Fortunately, the thirteen travelers on board were unharmed, but the effects were dramatic because the rail links were blocked over a large period of time due to the complexity of the rescue and restoration operations.

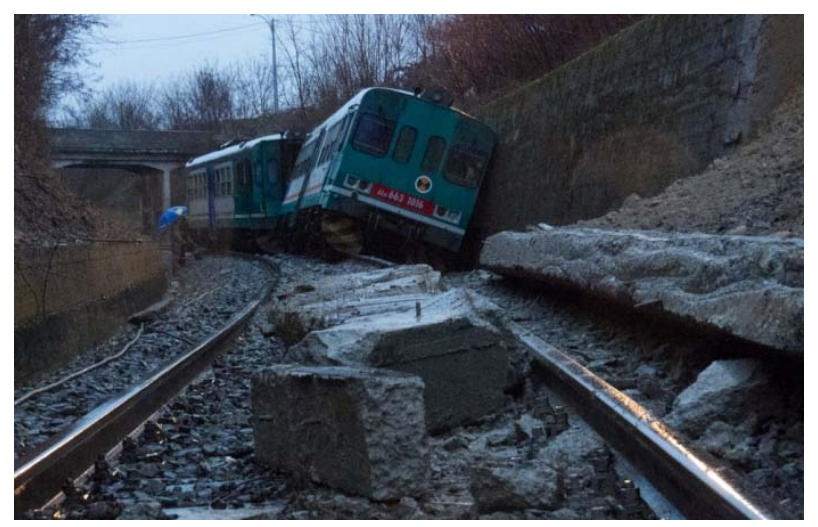

Figure 1. Disaster scenario of the railway system in Piedmont region.

The case illustrated above shows that, every time and anywhere, such events can happen and vehicles have to be equipped with a satellite-based tracking unit in order to know exactly where the train is located at the moment of a disaster. This bit of information is also a must to stop other trains traveling to the affected area. Indeed, the effects for the mentioned case become catastrophic if another train hits the one that is obliged to stop. A real-time positioning is also required for transport of critical goods. Moreover, since surveillance of data (temperature, humidity, etc.) within each wagon is also necessary during the travel, data on local conditions should also be collected.

\subsection{Global Navigation Satellite Systems: A Brief Overview}

Consolidated and available technologies for a precise 
location are based on Global Navigation Satellite Systems (GNSS). The navigation systems provided by engineering advances in satellite technologies were initially the NAVSTAR GPS (Navigation System with Time and Ranging - Global Positioning System) developed and maintained by the U.S. Department of Defense and Transportation, and the Russian Global Navigation Satellite System GLONASS (Globaluaya Navigatsionnaya Sputnikovaya Sistema) ${ }^{[7]}$. Although both are national military systems, they were available for use by the international private and commercial communities $^{[8,9]}$.

Recently a Chinese system (BeiDou) was completed as well as two of them designed and implemented by India, the Indian Regional Navigational Satellite System (IRNSS) and Japan, the Quasi Zenith Satellite System (QZSS), respectively ${ }^{[10,11]}$.

In Europe, concern was voiced about dependence on a foreign military system and thus plans for a civilian global navigation satellite system were put in place.

GPS employs two fundamental observables for positioning and navigation, the pseudo-ranges and the carrier-phase. The first one is a measure of the distance between the satellite at the time of transmission and the receiver when receives the signal. When used in instantaneous stand-alone mode, one obtains an accuracy of $10-20 \mathrm{~m}$ after the removal of selective availability. The second one can be used to determine ranges with millimetric accuracy, provided the integer ambiguity problem is solved ${ }^{[12-14]}$.

The system was divided in an overlay for the existing systems (GNSS-1), and a completely autonomous one with a separate space segment (GNSS-2). Further, the drop of equipment prices over the past ten years led to an enormous growth in the number of GPS users and new applications emerged such as car navigation systems, fleet management, aircraft approach, bridge deformation monitoring, and the navigation of agricultural field machinery. These multiple applications increased the necessity to improve accuracy, availability, and integrity in the systems.

Currently, Differential GNSS (DGNSS) represents a solution for these user requirements, where correctional data are emitted from a reference system placed in a known position and sent to a receiver. In differential positioning, a minimum of two receivers, named Base Station and Rover, are involved. The Base Station occupies a known position during the session, and the determination of the Rover related to the base rep- resents the target of the work. Both receivers observe the same constellation of satellites at the same time, and since the base position is known, corrections may be generated in order to improve the solution at the Rover, as shown in Figure 2.

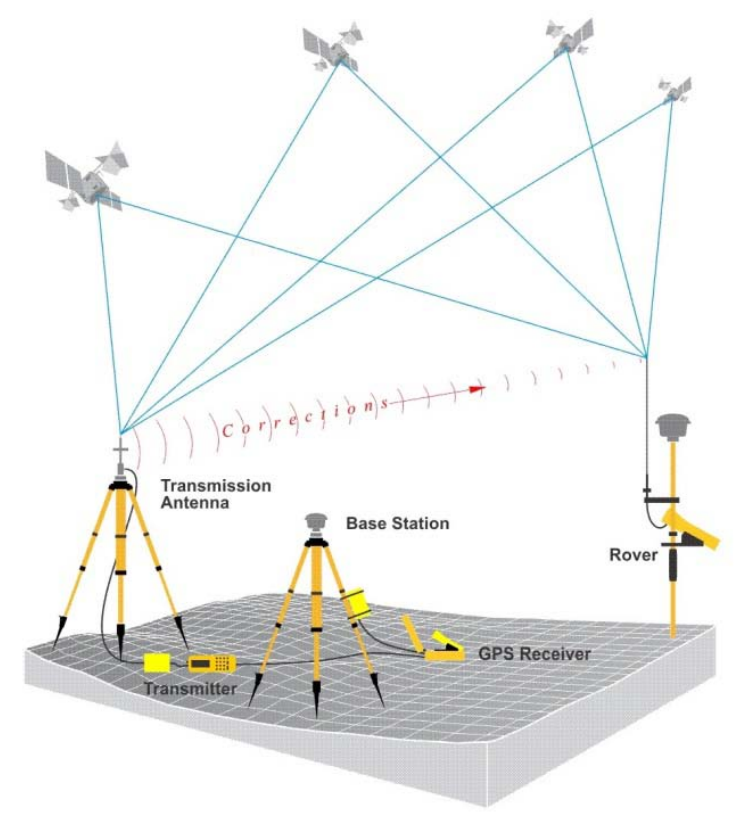

Figure 2. Differential GNSS with RTK Positioning.

The GPS signal is affected by a number of error sources including orbital and atmospheric propagation errors $^{[15]}$. An improvement in the GPS performance can take the form of enhancements in the geometry of data sources (satellites), measurement quality, or both. It is wellknownthat GPS is also unable to measure low velocity, therefore, radar odometer or wheel sensors can be adopted in order to measure speed and improve the position accuracy in transport applications, as fully described in Section Four.

\section{The European Challenge}

The strategy for the European involvement in the next generation of Global Navigation Satellite Systems (GNSS-2) consists of an independent navigation space-segment and respective ground infrastructure under the designation of GALILEO ${ }^{[16-18]}$. The aim of this decision is to overcome the unacceptable dependency on third-party states, which is directly linked to the sovereignty issues emerging if Europe uses foreign-controlled navigation systems for critical security applications. Moreover, the possible introduction of user-fees for GPS infrastructure and services has to be considered as well as the drawback of having 
no access to the technical development of the system, in order to ensure the participation of European industry in the fast-growing market of satellite navigation.

The first two satellites, which are part of the operational system, were launched in October 2011 and are denoted as ProtoFlight Model (PFM) and Flight Model 2 (FM2), reported as E11 and E12 in RINEX observation files. The second pair of IOV satellites (FM3 and FM4) was launched on October 2012, and started signal transmission in December of the same year. All IOV satellites are equipped with two Passive Hydrogen Masers (PHMs) and two Rubidium Atomic Frequency Standards (RAFSs) which are used as quality indicator for the orbits.

As of December 2015, GALILEO counts 12 satellites in orbit, but the implementation of the complete 30 -satellite constellation (24 operational and 6 active spares) continues to be postponed so that the Full Operational Capability (FOC) is currently planned for year 2020.

\subsection{Simulations of Realistic Scenarios}

The delay of the European release, which is expected to have an open architecture, completely independent of the current navigation systems, forced the consortium involved in the research activity reported in this paper to use the GPS signals for testing the implemented prototype. Nevertheless, laboratory tests with the help of a multi-GNSS simulator provided by Spectracom Corp. (GSG-62) were also carried out at the University of Pavia in order to design, develop, and validate GALILEO-ready solutions, i.e., solutions with functionalities able to exploit the features of the innovative satellite system ${ }^{[19]}$.

The opportunity to use systems that simulate satellite constellations arises from the need for all the providers of GNSS receivers to test their devices and ensuring or improving their capabilities in term of accuracy and reliability. A GSG-62 multi-channel simulator generates the same RF signals that are broadcast by either GALILEO or GPS navigation satellites. Indeed, it offers multiple simulation of constellation with a base of 32 channels, expandable up to 64, and allows the building of real time trajectories within the StudioView $^{\mathrm{TM}}$ software environment. In this way, the GPS signals are used to check the position accuracy of the receiver chosen by the consortium for the SPARTACUS project (Ellipse-N) ${ }^{[20]}$. Simultaneously, the trajectory provided by the GALILEO satellites may be used to upgrade the system in the acquisition and transmission of the message from the data collector to a control center. After that, the path is compared with the GPS reference track within Google Earth through the NMEA standard format ${ }^{[21]}$, as shown in Figure 3.

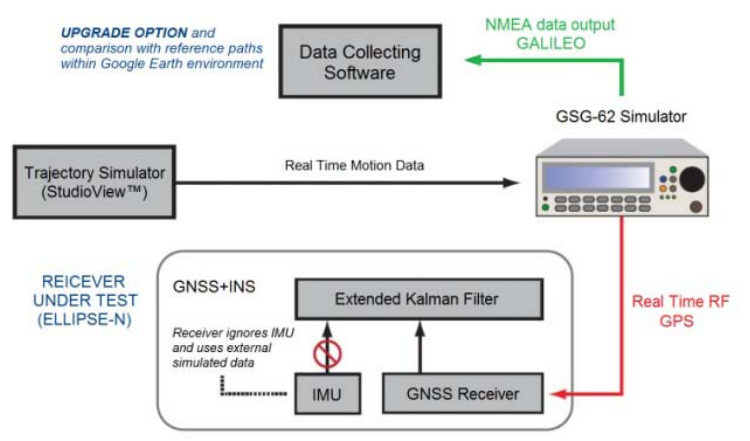

Figure 3. Scenario of a real time trajectory simulation for laboratory test.

The experimental results proposed in this section aim to estimate the value of the upgrade option of the software expected for the collection of positioning data with the upcoming European Satellite System (GALILEO) in the urban canyon of Pavia, Italy. For this reason, two different trajectories respectively with 4 and 8 satellites are carried out, both starting from the Department of Civil Engineering and Architecture (DICAr), up to the Campus Aquae, placed nearby (Via Cascinazza 15) and the Railway Station (located in Via Brichetti 40), respectively. The latitude and longitude can be extracted by NMEA strings and imported in Google Earth to provide a visual comparison of the performed track (Figure 4A). Azimuth and elevation are also handled in Matlab ${ }^{\circledR}$ software environment in order to offer a GALILEO satellites view (Figure 4B).

The described approach was essential first for the hardware integration required by the design of the proposed tracking system. Moreover, it made the software updating possible in two aspects: (i) the local storage of the needed positioning information, and (ii) its sending to the operative center using a low-power wireless communication. Once the GALILEO signal simulator has been set for the transfer of NMEA sentences, to configure the acquisition code for the oncoming European Satellite Navigation System was eventually done.

\section{The SPARTACUS Solution}

GALILEO intends to provide a more robust positioning capability enhancing the adoption of satellite 


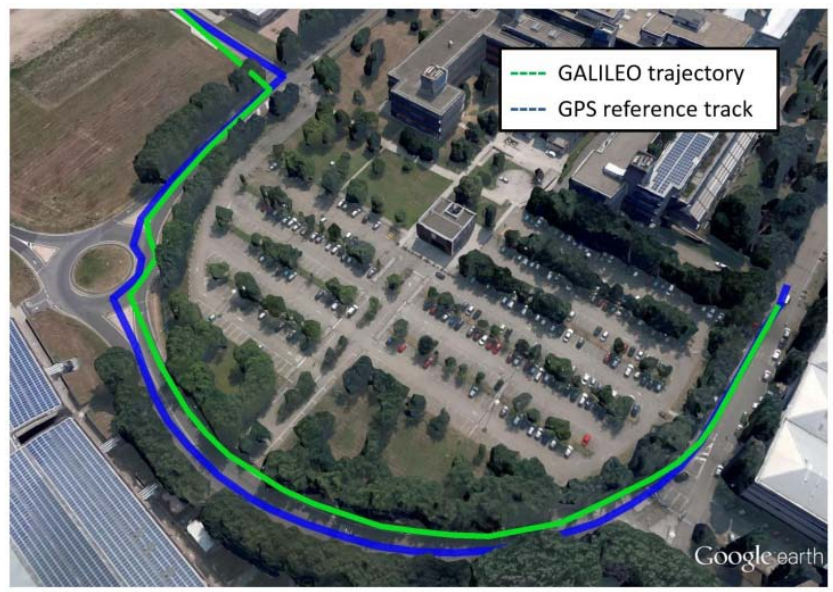

(A)

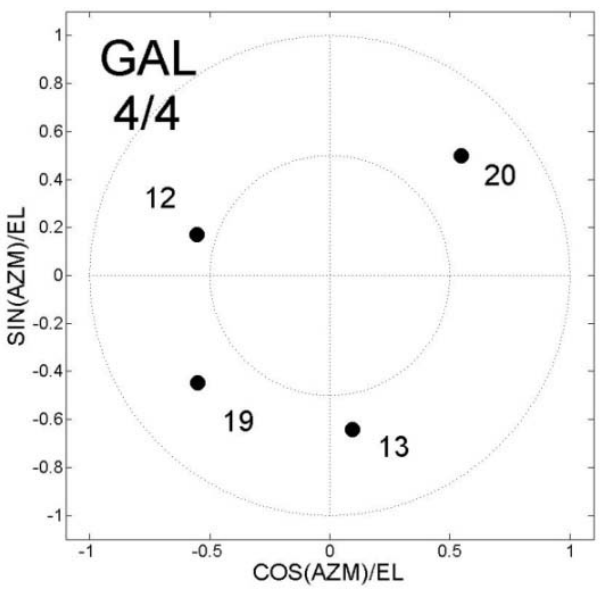

(B)

Figure 4. Trajectories comparison in Google Earth (A) and GALILEO satellites view (B).

technologies for solutions that requires signal continuity and integrity. The impact is on various application areas, including emergency and disaster management, rescue tasks and location-based services supporting responders in critical operations. The ongoing SPARTACUS project started to develop new EU-specific services for the following three application areas: (i) critical transport assets in case of major failure of existing networks, (ii) flow of relief support goods from the sending side to the receiving place, and (iii) first responders ensuring their safety in crisis management operations.

These application areas are in line with the overall scope of the project, that is to design, realize, test and validate GALILEO-ready tracking/positioning solutions in simulated and real world for critical asset tracking and crisis management. The output is the definition of system architectures and components with features targeted to satisfy the needs of the users involved.

The solutions designed are based on providing precise localization of goods in harsh environment during absence of signal (canyons, tunnels etc.), guaranteeing effective local storage with a communication point from multiple tracking units to a remote site, and ensuring communication network over the failure of the existing terrestrial infrastructures in disaster situations.

The research activity carried out in this paper is focused on the positioning of all components concerning the freight transport, such as containers, wagons and locomotives (Figure 3). Particular attention is paid to the data transmission from each GNSS based tracking units to the collecting centre, since the conventional cabling method suffers problems due to inflexible installation and negative impact on the transportation vehicle.

As shown in Figure 5, the local wireless network for transport application consists of several GNSS sensors integrated with a ZigBee Node through RS232 serial port (tracking units), and each of them communicates with the collecting unit integrated with a ZigBee Base Station. The GNSS sensors acquire the wagons/container position from the GNSS satellites signal with a frequency of $1 \mathrm{~Hz}$ and send the position data to a centralized unit, which is located on board the locomotive through the multi-hop ZigBee mesh network. Finally, the collecting unit is linked to a remote centre through a satellite backhauling device (communication unit), avoiding the existing terrestrial communication infrastructure.

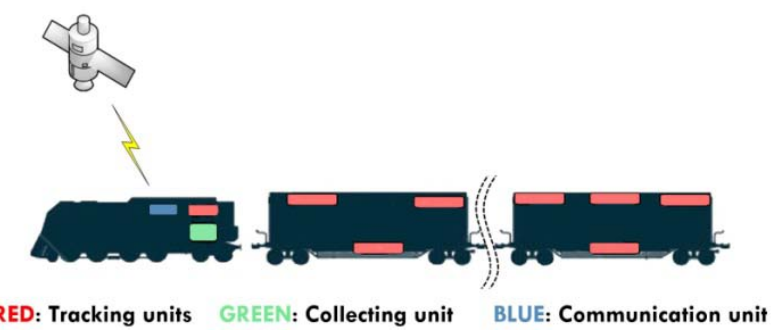

Figure 5. Case under study: transport application.

\subsection{Features of a Single Board Tracking Unit}

Reliability, accuracy, low cost and low power are the main qualities required by a positioning system for the infrastructure monitoring of a smart city. The selected devices for the development of a single board tracking unit consist of an inertial sensor with internal GNSS 
receiver, a credit-card sized computer, and a GBAN ZigBee Wireless Data Transmission Device. The specific functions for each component of the tracking unit are listed in Table 1.

Table 1. Technical specifications and functions of the tracking unit

\begin{tabular}{|c|c|}
\hline Hardware & Function \\
\hline Ellipse-N & $\begin{array}{l}\text { Exploit INS capabilities without navigation } \\
\text { satellites signal }\end{array}$ \\
\hline GNSS antenna & $\begin{array}{l}\text { Acquire GNSS positioning data with quality } \\
\text { field (accuracy/precision) }\end{array}$ \\
\hline Raspberry Pi 2 & $\begin{array}{l}\text { Collect positioning information to allows local } \\
\text { storage and post-processing }\end{array}$ \\
\hline PiFace Shim RTC & $\begin{array}{l}\text { Provide a continuous timing through an external } \\
\text { local clock }\end{array}$ \\
\hline External sensors & $\begin{array}{l}\text { Supply information on temperature, humidity, } \\
\text { and limit switch }\end{array}$ \\
\hline GBAN ZigBee & $\begin{array}{l}\text { Send information to the collecting unit using } \\
\text { Wireless Transmission Devices }\end{array}$ \\
\hline
\end{tabular}

Therefore, in order to achieve data post-processing, the system has to be equipped with a data collector (Raspberry Pi) able to manage a local storage of the information to be transmitted to the remote control. Figure 6 shows all the hardware components for the final prototype used for the testing activity. The Raspberry Pi 2 is based on the Broadcom BCM2835 system on a chip (SoC). It is supplied with 512MB of RAM, four Universal Serial Bus (USB), and Micro Secure Digital (Micro SD) sockets for boot media and persistent storage. Figure 6 also shows the Ellipse $\mathrm{N}$ with its GNSS antenna provided by SBG Systems, which is a miniature sensor with high-performance Micro Electro-Mechanical Systems (MEMS). It also includes an Inertial Measurement Unit (IMU), an on-board enhanced Extended Kalman Filter (EKF),

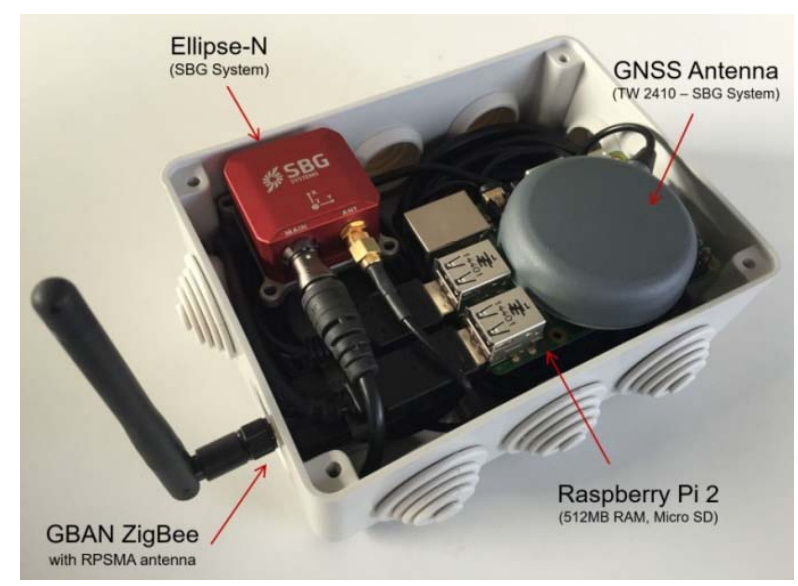

Figure 6. Hardware components of the positioning system. and supports standard protocols such as the NMEA0183 for direct integration into existing applications $^{[22]}$.

Once the positioning data are correctly stored inside the memory of the data collector, the information is sent by ZigBee devices to a collecting unit placed on board the locomotive in order to be transmitted via satellite to a remote centre for monitoring in real-time goods and vehicles. Among those available ZigBee technology offers a great trade-off in terms of power, range, data rate, and security. The ZigBee organizes multi-hop network and imposes that two devices exchange data depending on other intermediate forwarding devices. Further, the nature of this system requires that each device perform a specific networking function that determines its role within the network. The developed network consists of a Coordinator, Router and End device nodes, but the device type does not restrict the type of application that may run on the particular device because it is usually determined at compile-time ${ }^{[23]}$.

\subsection{System Performance in Harsh Environment}

The SPARTACUS tracking system is designed to work anywhere in the same way, regardless of GNSS signal availability. Currently, Europe's Transport positioning applications are based on Radio-Frequency Identification (RFID) and European Rail Traffic Management System (ERTMS). For this kind of applications the absence of signal coverage cannot occur because the position is determined by a set of precise points located along train paths.

SPARTACUS proposes to determine the position independently from external infrastructures ensuring low cost and dead reckoning capabilities. Indeed, the adopted sensor integrates an Extended Kalman Filter (EKF) to estimate the real time position, velocity and orientation of the train. Figure 7 shows the acceleration response produced by the three-axial Crossbow $\mathrm{CXL} 1 \mathrm{~F}^{[24]}$ and the EKF integrated into the inertial navigation system.

When aiding data such as GPS positions are available, the EKF corrects the current state preventing drifts, but when the measurement is not received, the estimation error tends to increase.

In this case, the system only relies on the inertial sensor in order to continuously track position, velocity and orientation. Since cost constraints are strict within the project, MEMS technology represents the most suitable solution, but sometimes the position drift can 

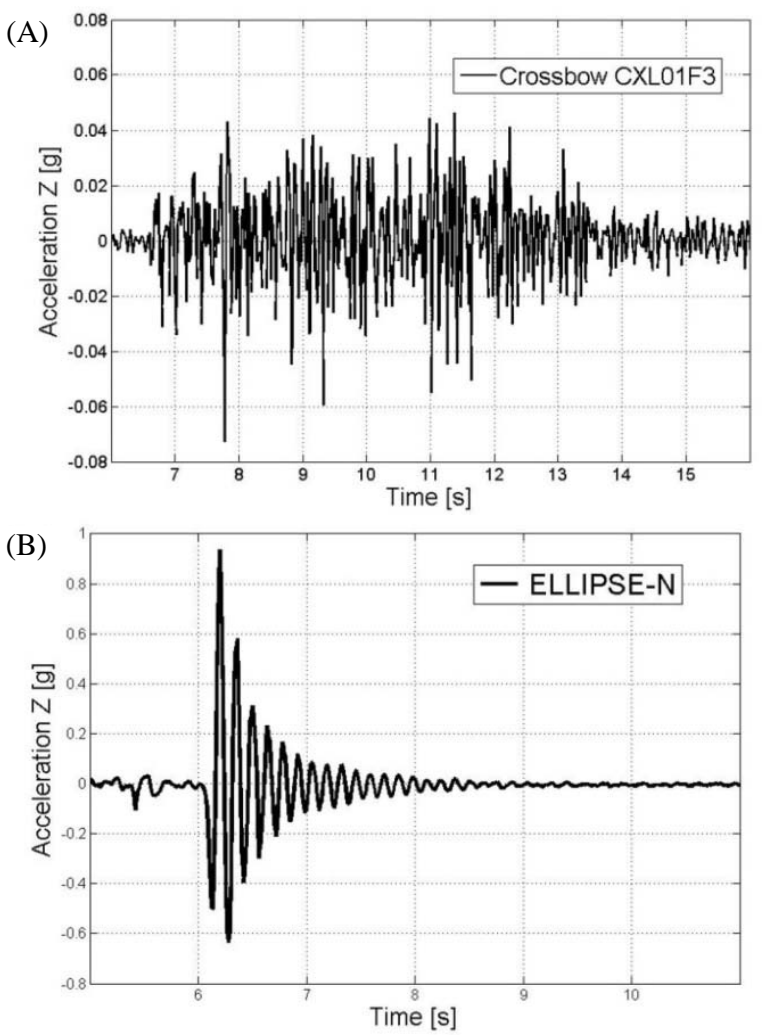

Figure 7. Crossbow accelerations (A) and EKF effect on the adopted sensor (B).

reach meters after just few seconds of free inertial navigation. For this reason, additional information to maintain proper accuracy are needed, even during long term GNSS drop outs. An odometer (GSS25 provided by Pegasem Messtechnik GmbH) is adopted ${ }^{[25]}$. The GSS25 has to be placed on the wheel of the vehicle and can be used to measure its average velocity when GNSS are not available. The odometer aiding reduces the position drift. The sensor can be simply added at the head of the train. Possible performance differences are explained by avoiding a thermal calibration on wagons and containers in order to save production time and cost.

\section{Experimental Data Set}

The field tests reported in this section cover the area around the urban canyon of Pavia (Italy) in order to ensure anytime/anywhere the positioning accuracy of the adopted system. Because it is designed to transport applications, the navigation system has to satisfy requirements for the urban environment. The precision of the signal can be mined by the characteristics of the urban areas, where physical restrictions are imposed such as buildings, narrow streets, tunnels or bridges, and for this reason is necessary to validate also the system capabilities in dead reckoning mode ${ }^{[26]}$.

The tracking unit is placed inside a car to reply the train motion profile and the entire system is supplied directly from the power supply of the vehicle. The paths with GNSS satellite signal coverage are repeated detaching the external GNSS antenna during the test. In this way, the satellite signal cannot be received by the sensor and the condition of a train inside a tunnel is obtained.

The two parameters used to measure the position accuracy of the system are the Average Positioning Error $(\mu)$ and its Standard Deviation $(\sigma)$, expressed in Equation 1.

$$
\mu=\sum_{i=1}^{N} \frac{x_{i}}{N} \quad \sigma=\sqrt{\sum_{i=1}^{N} \frac{\left(x_{i}-\mu\right)^{2}}{N-1}}
$$

where $x_{i}$ is the i-th positioning error, and $N$ is the total number of data. The robustness of the entire system has to be checked in case of disturbances or whatever changing of boundary conditions for very long period as well. Table 2 summarizes the results from the field test sessions carried out at several speeds in harsh environments.

Table 2. Experimental results obtained for the field test sessions

\begin{tabular}{ccccc}
\hline Set & $\mu(\mathrm{m})$ & $\sigma(\mathrm{m})$ & $\mathrm{v}_{\mathrm{m}}(\mathrm{m} / \mathrm{s})$ & $\begin{array}{c}\text { Positioning error } \\
\text { after 60s }(\mathrm{m})\end{array}$ \\
\hline $\mathrm{T} 01$ & 1.172 & 0.788 & 8.30 & 0.320 \\
$\mathrm{~T} 02$ & 2.924 & 2.505 & 9.70 & 4.019 \\
$\mathrm{~T} 03$ & 4.240 & 3.564 & 15.0 & 2.030 \\
$\mathrm{~T} 04$ & 8.406 & 5.675 & 15.8 & 2.560 \\
$\mathrm{~T} 05$ & 0.996 & 0.597 & varying & 0.219 \\
\hline $\mathrm{T} 06$ & 6.670 & 6.212 & 20.0 & 13.34 \\
$\mathrm{~T} 07$ & 1.710 & 0.939 & 21.2 & 3.554 \\
$\mathrm{~T} 08$ & 9.546 & 4.069 & 27.8 & 17.75 \\
$\mathrm{~T} 09$ & 7.271 & 5.498 & 30.5 & 11.53 \\
$\mathrm{~T} 10$ & 3.264 & 1.144 & varying & 5.259 \\
\hline
\end{tabular}

The tests from T01 until to T05 are carried out in the urban environment, whilst those from T06 to T10 are addressed to the extra-urban one. The tracking system behavior is affected by the density of surrounding trees and buildings. The results in Table 2 are computed only on the time window when the GPS antenna is detached. The wished positioning error threshold is widely fulfilled in all of them, provided the GSS25 sensor is connected to the receiver. Collected data are also imported within Matlab ${ }^{\circledR}$ software 
environment where, after one minute from the detachment moment, accuracy is investigated by means the positioning error (Figure 8).

The case studies reported in Figure 8 show the odometer impact when stops ( 3 in the tests) are included along the trajectory without GNSS satellite signal coverage.

At last, the data integrity of the data acquisition software was investigated in order to check the performance of the described system. The correctness of the message acquired by the tracking unit is achieved through the percentage of the uncorrupted NMEA sentences, and a detailed analysis demonstrated that they are totally stored into the memory of the data collector.

\section{Conclusions}

The first 24 hours of an incident are the most critical in view of saving human lives. This aspect is crucial in the urban design of a smart city.

Past experiences proved that the availability of real time information is necessary to promptly inform the rescue teams and to protect the people suffering the natural disaster. This research activity details the development of a portable and modular system to pro-
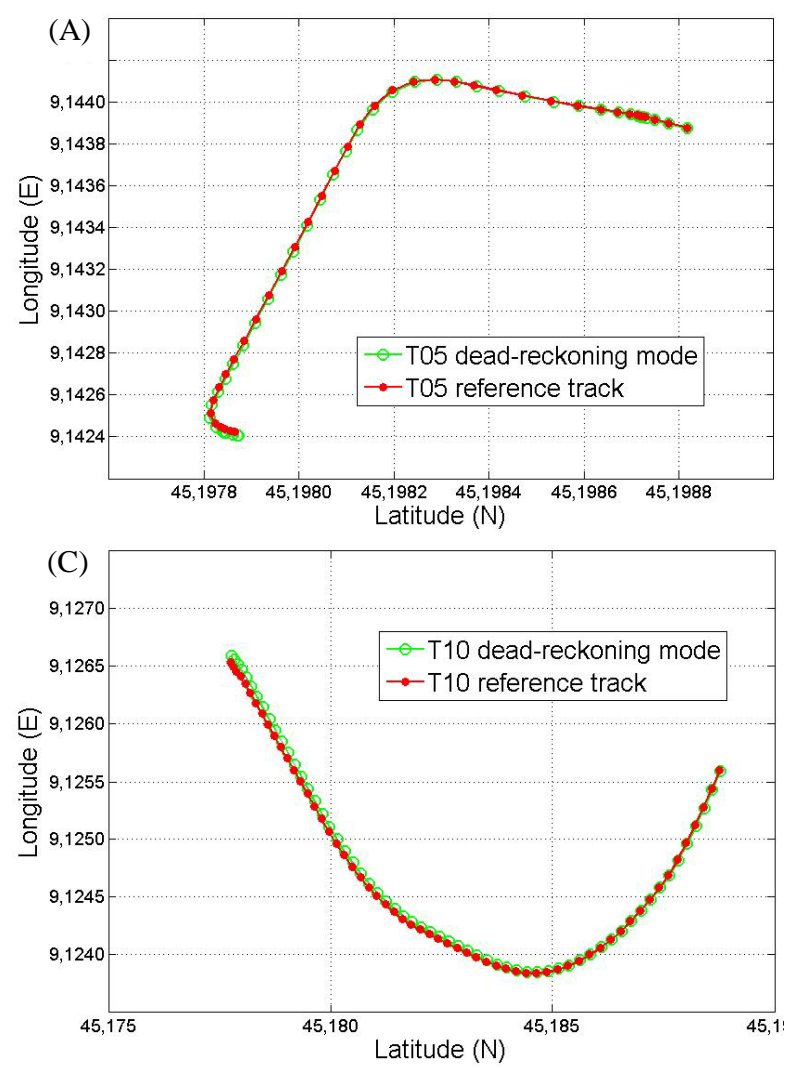

Figure 8. Case studies (A-C) and positioning error (B-D). vide non-terrestrial based crisis management support. Improving efficiency and saving costs are the two main issues. One of the most important services that can be offered to the citizen of a smart city is the capability of an independent network with satellite backhaul, which is always able to connect, even independently of availability of local infrastructure, providing access to large user groups (i.e. rescue teams as well as victims). The result of a broad experimental campaign is here reported to validate the inertial platform of the system in order to provide dead zone reckoning. Indeed, GNSS dead zones such as valleys, tunnels, and building interiors have to be overcome for critical assets and crises. Moreover, the involved technology envisages future use of the GALILEO signal, exploiting the important benefit of the integrity information which is of highest importance in the Public Protection and Disaster Relief (PPDR) scenarios. It goes much beyond the possibilities of current GPS offer.

\section{Conflict of Interest and Funding}

No conflict of interest was reported by the authors. The research leading to these results has received
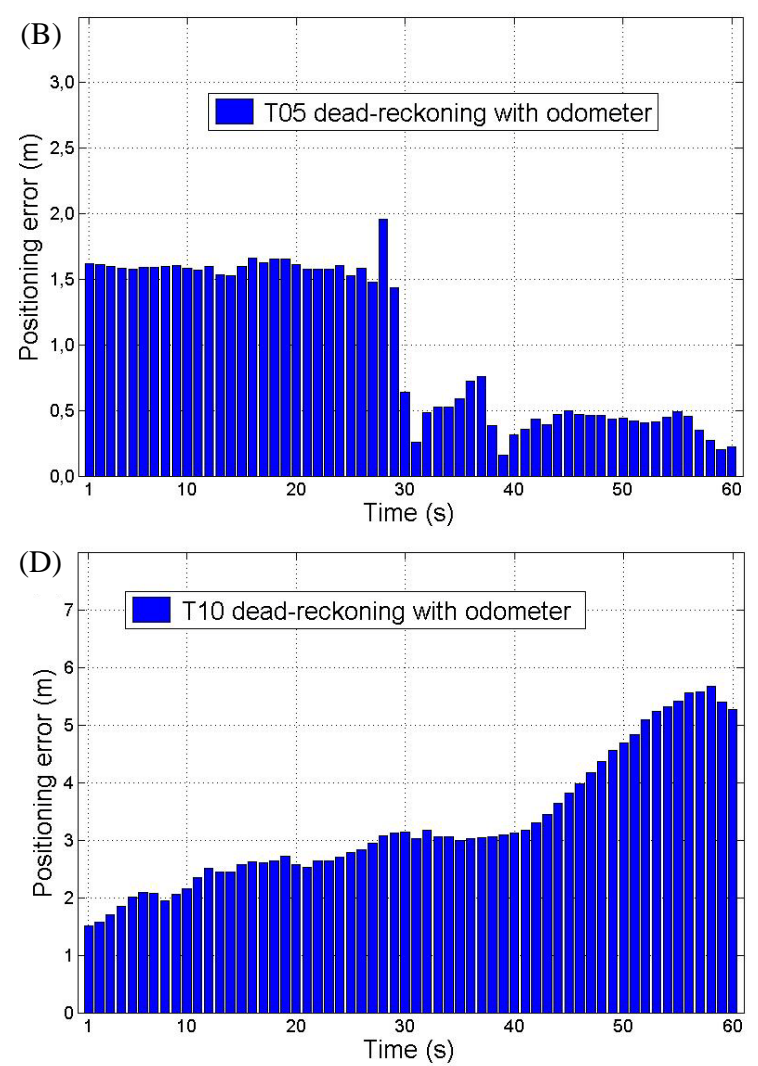
funding from the European Union Seventh Framework Programme (FP7/2007-2013) under grant agreement $n^{\circ}$ [313002].

\section{References}

1. Casciati F, Casciati S, Faravelli L, et al. 2015, Development of an Outline Detection Tool in QGIS environment for risk mitigation applications, Journal of Smart Cities, vol.1(1): 59-67.

2. D’Alessandro A, Ubertini F, Laflamme S, et al. 2015, Towards smart concrete for smart cities: Recent results and future application of strain-sensing nanocomposites, Journal of Smart Cities, vol.1(1): 3-14. https://doi.org/10.18063/JSC.2015.01.002.

3. Komninos N, Bratsas C, Kakderi C, et al. 2015, Smart City Ontologies: Improving the effectiveness of smart city applications, Journal of Smart Cities, vol.1(1): 31-46. https://doi.org/10.18063/JSC.2015.01.001.

4. Spartacus Project, 2016, Satellite Based Asset Tracking for Supporting Emergency Management in Crisis Operations (SPARTACUS), viewed October 29, 2016, $<$ http://www.spartacus-project.eu/home>

5. Casciati F, Casciati S, Chen Z-C, et al. 2014, Development of a eeliable wireless GNSS sensor network. Proceedings of EWSHM 2014, 7th European Workshop on Structural Health Monitoring, Nantes, France.

6. Ochieng W Y and Sauer K, 2002, Urban road transport navigation: performance of the global positioning system after selective availability. Transportation Research Part C, vol.10(1), 171-187.

https://doi.org/10.1016/S0968-090X(02)00008-6.

7. Lechner W and Baumann S, 2000, Global navigation satellite systems, Computers and Electronics in Agriculture, vol.25(1): 67-85.

https://doi.org/10.1016/S0168-1699(99)00056-3.

8. Leick A, 1995, GPS Satellite Surveying, $2^{\text {nd }}$ edn, John Wiley \& Sons, Inc., USA.

9. Parkinson B W, Spilker J J Jr, Axelrad P, et al. 1996, Global Positioning System, Volume I, American Institute of Aeronautics and Astronautics, Inc., USA.

10. Odijk D, Nadarajah N, Zaminpardaz S, et al. 2016, GPS, Galileo, QZSS and IRNSS differential ISBs: estimation and application, GPS solutions, in press. https://doi.org/10.1007/s10291-016-0536-y.

11. Quan Y, Lau L, Roberts G W, et al. 2016, Measurement signal quality assessment on all available and new signals of multi-GNSS (GPS, GLONASS, Galileo, BDS, and QZSS) with real data, Journal of Navigation, vol.69(2): 313-334. https://doi.org/10.1017/S0373463315000624.

12. Vittuari L, Gottardi G and Tini M A, 2015, Monumentations of control points for the measurement of soil vertical movements and their interactions with ground water contents, Geomatics, Natural Hazards and Risk, vol.6(5-7): 439-453.

https://doi.org/10.1080/19475705.2013.873084.

13. Zanutta A, Vittuari L and Gandolfi S, 2008, Geodetic GPS-based analysis of recent crustal motions in Victoria Land (Antarctica), Global and Planetary Change, vol.62(1): 115-131. https://doi.org/10.1016/j.gloplacha.2008.01.001.

14. Abbondanza C, Altamimi Z, Sarti P, et al. 2009, Local effects of redundant terrestrial and GPS-based tie vectors in ITRF-like combinations, Journal of Geodesy, vol.83(1): 1031-1040. https://doi.org/10.1007/s00190-009-0321-6.

15. Fuggini C, 2010, Using satellites systems for structural monitoring: accuracy, uncertainty and reliability. Ph.D. dissertation, University of Pavia, Pavia, Italy.

16. Hackel S, Steigenberger P, Hugentobler U, et al. 2015, Galileo orbit determination using combined GNSS and SLR observations. GPS Solutions, vol.19(1): 15-25. https://doi.org/10.1007/s10291-013-0361-5.

17. Fernández-Hernández I, Rijmen V, Seco-Granados G, et al. 2016, A navigation message authentication proposal for the Galileo Open Service, Navigation: Journal of the Institute of Navigation, vol.63(1): 85-102. https://doi.org/10.1002/navi.125.

18. Borio D, Gioia C, 2016, Galileo: the added value for integrity in harsh environments, Sensors, vol.16(1): 1-23. https://doi.org/10.3390/s16010111.

19. Spectracom, 2013, GSG-5 / 6 Series GPS and GLONASS Simulator - User Manual, NY, USA.

20. Guinamard A, 2014, Ellipse AHRS \& INS - High Performance, Miniature Inertial Sensors User Manual, SBG Systems, Rueil-Malmaison, France.

21. Trimble, 2004, NMEA-0183 Messages Guide for AgGPS Receivers, Trimble Navigation Limited, Kansas, USA.

22. Casciati F, Casciati S, Chen Z-C, et al. 2015, Collecting data from a sensor network in a single-board computer. 11th International Conference on Damage Assessment of Structures (DAMAS 2015). Journal of Physics: Conference Series, vol.628(1):1-8.

23. Texas Instruments, 2009, CC2530 Software Examples User's Guide, Texas Instruments Incorporated, Dallas, Texas, USA.

24. Crossbow Technology Inc, 2016, CXL-LF Series - High Sensitivity Accelerometers, Document Part: 6020-000201 Rev E, San Jose, California, USA.

25. Pegasem Messtechnik GmbH, 2015, GSS Series Ground Speed Sensor - User Manual V 1.38, Noerdlinger, Germany.

26. Casciati S, Chen Z-C, Faravelli L et al. 2016, Synergy of Monitoring and Security. Smart Structures and Systems, vol.17(5): 743-751.

https://doi.org/10.12989/sss.2016.17.5.743. 